\title{
The Two Extra Element Theorem
}

\author{
R.D. Middlebrook \\ California Institute of Technology
}

Abstract

The Two Extra Element theorem (2EET), a logical extension of the single EET, enables a transfer function to be assembled, essentially in final form, from separate calculations on a circuit model that is simpler by the absence of two elements. The 2EET provides not only a potentially shorter, simpler, and easier analysis method, but also contributes a Low-Entropy Expression in which the ordering and grouping of terms contains useful design-oriented information. As an example, the $2 E E T$ is employed in design-oriented analysis of a bridged-T filter.

The (single) Extra Element Theorem (EET) [1] states that any transfer function of a linear system (for example, a gain) can be expressed in terms of its value when a given "extra" element is absent (the "reference gain"), and a correction factor involving the extra element $\mathrm{Z}$ and two driving point impedances (dpi's) $Z_{d}$ and $Z_{n}$ seen by the element.

One class of applications is when a system has already been analyzed and later an extra element is to be added to the model: the EET avoids the analysis having to be restarted from scratch. Another class of applications is when a system is to be analyzed for the first time: if one element is designated as "extra," the analysis can be performed on the simpler model in the absence of the designated element, and the result modified by the EET correction factor upon restoration of the "extra" element.

Applications in this second class can be considerably enhanced by an extension of the EET, the Two Extra Element Theorem (2EET), by which the effect of two simultaneously restored elements $\mathrm{Z}_{1}$ and $\mathrm{Z}_{2}$ can be determined by a correction factor involving dpi's seen by each extra element when the other is in a reference condition (open or short).

\section{Motivation for the EET and the 2EET}

The above descriptions of the EET and the 2EET are usually insufficient to inspire a student to use the theorems by his own choice. Motivation is built by emphasizing how much work is saved in getting the result, why the result is in a useful form, and how easy it is to use the theorems. A review of the simple Extra Element Theorem provides a convenient vehicle to illustrate these points.

A common situation is when a linear system model has been analyzed for a certain transfer function, perhaps with considerable mental effort, and later one wishes to add another element to the model. A typical example is when a capacitance needs to be added to account for a later observed pole in the frequency response. The usual approach is to rework the entire analysis from scratch which, of course, is even more work than it was the first time through.

Use of the EET avoids this repeat derivation by taking advantage of the result already obtained, and multiplying it by a correction factor that accounts for the extra element. With respect to Fig. 1, in which throughout this review of the single EET the

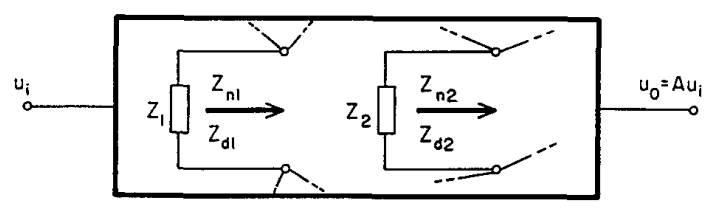

Fig. 1. Generalized linear system model for which any transfer function, for example a voltage gain $A$, is to be expressed in terms of its value when two "extra" elements $Z_{1}$ and $Z_{2}$ are absent, and a correction factor involving $Z_{1}$ and $Z_{2}$ and appropriate driving-point impedances.

element $\mathrm{Z}_{2}$ is absent (replaced either by an open or a short), the transfer function of interest is represented by the voltage gain $A \equiv v_{2} / v_{1}$, where $v_{1}$ is an independent source (the "input"), and $v_{2}$ is a corresponding dependent signal (the "output"). The EET expresses $A$ in the presence of an element $Z_{1}$ in terms of a reference gain multiplied by a correction factor:

$$
\mathrm{A}=\left.\mathrm{A}\right|_{\mathrm{Z}_{1}=\infty} \frac{1+\frac{\mathrm{Z}_{\mathrm{n} 1}}{\mathrm{Z}_{1}}}{1+\frac{\mathrm{Z}_{\mathrm{d} 1}}{\mathrm{Z}_{1}}}=\left.\mathrm{A}\right|_{\mathrm{Z}_{1}=0} \frac{1+\frac{\mathrm{Z}_{1}}{\mathrm{Z}_{\mathrm{n} 1}}}{1+\frac{\mathrm{Z}_{1}}{\mathrm{Z}_{\mathrm{d} 1}}}
$$

where $Z_{d 1}$ and $Z_{n 1}$ are two driving point impedances (dpi's) seen by the element $Z_{1}$. The dpi's are determined by replacement of $Z_{1}$ by a second independent source: $Z_{d 1}$ is the dpi seen by the second source when $v_{1}=0$ (single injection); $Z_{n l}$ is the dpi seen by the second source when $v_{1}$ is restored and the two independent sources are adjusted to null $v_{2}$ (null double injection). The principle of null double injection is discussed and illustrated in [1].

In the dual forms of the EET of Eq. (1), the reference gain is either $\left.A\right|_{Z}=\infty$ or $\left.A\right|_{Z_{1}=0}$ according to which represents the result already obtained before the extra element $Z_{1}$ is introduced. That is, there are dual forms of the theorem according to whether the extra element is to be introduced in parallel with an existing node pair, or in series with an existing branch. Obviously, there is a redundancy relation between the two reference gains and the two dpi's:

$$
\frac{\left.\mathrm{A}\right|_{\mathrm{Z}_{1}=0}}{\left.\mathrm{~A}\right|_{\mathrm{Z}_{1}=\infty}}=\frac{\mathrm{Z}_{\mathrm{n} 1}}{\mathrm{Z}_{\mathrm{d} 1}}
$$

To apply the EET, one need merely multiply the previously obtained reference gain by the correction factor involving the extra element itself and the two dpi's seen at the port to which the extra element is to be connected. This explains the principal benefit from use of the theorem: no calculation is ever done on the circuit model in the presence of the extra element. Instead, two calculations (the dpi's) are made on the original circuit model, the reference circuit, before the extra element is attached. While there is no guarantee that calculation of the two dpi's in the reference circuit is easier than calculation of the new transfer function with the extra element in place, this is usually the case.

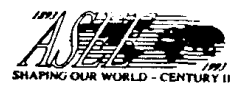

1991 Frontiers in Education Conference 
As discussed in [1], calculation of $\mathrm{Z}_{\mathrm{d} 1}$ is usually longer and more difficult than calculation of $Z_{n 1}$, in spite of the fact that the null double injection principle involved in $\mathrm{Z}_{\mathrm{l}}$ may be unfamiliar. Indeed, it may be preferable to bypass the direct calculation of $Z_{d}$ and instead calculate the other reference gain and find $\mathrm{Z}_{\mathrm{d} l}$ indirectly from the redundancy relation of Eq. (2). Either way, calculation of both dpi's is easy and straightforward.

Another benefit of the EET results from its being a LowEntropy Expression. A Low-Entropy Expression, discussed in [2], is one in which the various elements or terms are grouped, or ordered, in such a way that more design-oriented information is obtained than merely the single number for the "answer" when numerical values are substituted for the various elements.

The EET is a Low-Entropy Expression because its format immediately exposes the manner in which the extra element influences the result. That is, the effect of the extra element is determined by its value relative to the two dpi's. In some cases, this may be the most useful aspect of the theorem: how to choose a value for an extra element so that it does not significantly affect a previously designed transfer function. An example of this use is the development of design criteria for a line input filter to be added to an already designed regulated power supply [3]: the output impedance of the input filter represents the extra element, and the design criteria are that the input filter output impedance should be much less than the dpi's corresponding to the relevant transfer functions, of the original power supply, that are not to be significantly affected.

It was also discussed in [1] how the EET can be useful in another class of applications, for analysis of circuit models in which all elements to be incorporated are known at the outset. In this sort of "reverse" application, one element is designated "extra" and is omitted, to be replaced by either an open or a short. The omitted element is then restored by use of the EET. The potential value of this approach accrues from two of the benefits of the EET.

First, all three of the calculations (the reference transfer function and the two dpi's) are made on the reference circuit, in the absence of the extra element; that is, calculation of the required transfer function, for the original circuit, is never done directly, but is replaced by three separate calculations on the simpler reference circuit.

Second, the Low-Entropy format of the EET makes the result particularly simple and illuminating when the designated "extra" element is a reactance and the rest of the circuit, the reference circuit, is resistive: then, both dpi's are resistances, and the numerator and denominator of the EET correction factor directly give the zero and pole, respectively, due to the reactance.

A logical extension of this reasoning provides the motivation for development of the Two Extra Element theorem. Although it would be unlikely that one would want to modify a transfer function to account simultaneously for two previously unrecognized extra elements, there is considerable potential advantage to be obtained from a Low-Entropy Expression for a transfer function in which the influences of two designated elements are directly exposed, in terms of their dpi's. Then, the transfer function for the complete circuit could be assembled from the results of calculations made only upon a reference circuit that is simpler by the absence of two elements. The format would be even more useful, again, if the two designated extra elements are reactances and the reference circuit is resistive, since zeros and poles would immediately be apparent.

Some features of a 2EET can be anticipated. If the extra elements are designated $\mathrm{Z}_{1}$ and $\mathrm{Z}_{2}$, the result must be symmetric in the subscripts 1 and 2 , since obviously their allocation is arbitrary.

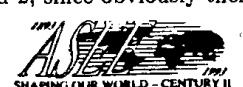

1991 Frontlers in Education Conference 703
If either $Z_{1}$ or $Z_{2}$ is zero or infinite, the 2EET must reduce to the corresponding single EET. Both numerator and denominator of the $2 \mathrm{EET}$, in general, must contain a term in the product $\mathrm{Z}_{1} \mathrm{Z}_{2}$ since, if $Z_{1}$ and $Z_{2}$ are similar reactances, there must be terms in $\mathbf{s}^{2}$, where $s$ is complex frequency. Consequently, in general when $Z_{1}$ and $Z_{2}$ are the only reactances, the zeros and poles of the transfer function will be in quadratic form.

\section{The 2EET}

The 2EET presents a transfer function of a linear system as the product of that transfer function when two designated elements $Z_{1}$ and $\mathrm{Z}_{2}$ are absent, and a correction factor involving the two designated elements and their appropriate driving point impedances $\mathrm{Z}_{\mathrm{n} 1}, \mathrm{Z}_{\mathrm{d} 1}$ and $\mathrm{Z}_{\mathrm{n} 2}, \mathrm{Z}_{\mathrm{d} 2}$. Figure 1 illustrates the structure, with the transfer function represented by the voltage gain $A \equiv v_{2} / v_{1}$.

Obviously, the gain A could be found by starting with its reference value when both $Z_{1}$ and $Z_{2}$ are absent, and then applying the single EET successively to incorporate first $Z_{1}$, say, and then $Z_{2}$. However, the result would not be very useful (it would be a HighEntropy Expression) because $Z_{n 2}$ and $Z_{d 2}$, the dpi's for $Z_{2}$, would contain $Z_{1}$ since $Z_{1}$ is already present when they are calculated. Thus, the benefits of a Low-Entropy Expression would be lost, since the influence of $Z_{1}$ would be obscured. Also, the symmetry with respect to $Z_{1}$ and $Z_{2}$ would not be apparent.

What is needed is to express $Z_{n 2}$ and $Z_{d 2}$ in terms of their values when $Z_{1}$ is absent, so that $Z_{1}$ again becomes explicit in the result. The derivation of the $2 E E T$, relegated to the Appendix, is done by following precisely this derivation sequence, so that the result is expressed in a Low-Entropy form, symmetric in $\mathrm{Z}_{1}$ and $\mathrm{Z}_{2}$ involving only the dpi's for one element when the other element is absent.

For the single EET, there are two forms in Eq. (1) depending upon whether the reference gain corresponds to the extra element being infinite (open) or zero (short). For the 2EET, there are three forms, corresponding to the three combinations of reference circuit: both extra elernents infinite, both zero, one of each. The three forms are:

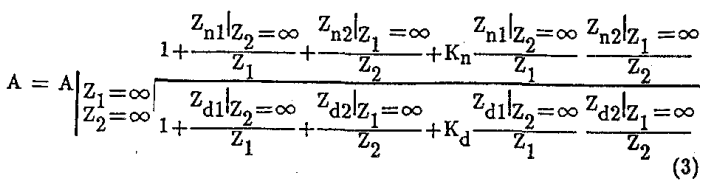

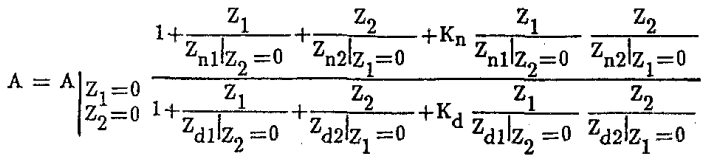

$$
\begin{aligned}
& 1+\frac{\mathrm{z}_{\mathrm{n} 1} \mathrm{z}_{2}=0}{\mathrm{Z}_{1}}+\frac{\mathrm{z}_{2}}{\mathrm{z}_{\mathrm{n} 2} \mathrm{z}_{1}=\infty}+\frac{1}{\mathrm{~K}_{\mathrm{n}}} \frac{\mathrm{Z}_{\mathrm{n} 1} \mathrm{Z}_{2}=0}{\mathrm{Z}_{1}} \frac{\mathrm{z}_{2}}{\mathrm{z}_{\mathrm{n} 2} \mathrm{z}_{1}=\infty}
\end{aligned}
$$

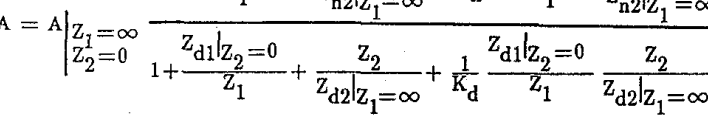

where

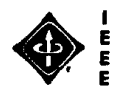




$$
\begin{gathered}
\mathrm{K}_{\mathrm{n}} \equiv \frac{\mathrm{z}_{\mathrm{n} 1} \mid \mathrm{z}_{2}=0}{\mathrm{z}_{\mathrm{n} 1} \mid \mathrm{z}_{2}=\infty}=\frac{\mathrm{z}_{\mathrm{n} 2} \mathrm{z}_{1}=0}{\mathrm{z}_{\mathrm{n} 2} \mathrm{z}_{1}=\infty} \\
\mathrm{K}_{\mathrm{d}} \equiv \frac{\left.\mathrm{z}_{\mathrm{d} 1}\right|_{2}=0}{\mathrm{z}_{\mathrm{d} 1} \mid \mathrm{z}_{2}=\infty}=\frac{\mathrm{z}_{\mathrm{d} 2} \mathrm{z}_{1}=0}{\mathrm{z}_{\mathrm{d} 2} \mathrm{z}_{1}=\infty}
\end{gathered}
$$

Although these expressions may appear somewhat formidable, they are actually Low-Entropy in that the format of the correction factor exposes many valuable features.

First, it is seen that the anticipated results are confirmed: each 2EET version is symmetric in $Z_{1}$ and $Z_{2}$; if either $Z_{1}$ or $Z_{2}$ is absent (that is, has its reference value infinity or zero), the 2EET reduces to the single EET; both numerator and denominator contain terms in the product $Z_{1} Z_{2}$. Of course, the fourth reference combination $\mathrm{Z}_{1}=0, \mathrm{Z}_{2}=\infty$ possesses a redundant result since it can be obtained from Eq. (5) by interchange of subscripts, and in any case is unnecessary since the subscript designations are arbitrary.

It may be noted that in each version of the 2EET the extra elements $Z_{1}$ and $Z_{2}$ are each normalized to a dpi calculated for the other extra element in the same reference condition as for the corresponding reference gain. For example, in Eq. (5), the reference gain corresponds to $\mathrm{Z}_{1}=\infty, \mathrm{Z}_{2}=0$, so the $\mathrm{Z}_{1}$ and $\mathrm{Z}_{2}$ terms in the correction factor are normalized to dpi's for $\mathrm{Z}_{2}=0$ and $\mathrm{Z}_{1}=\infty$, respectively. Hence, use of any version of the $2 \mathrm{EET}$ requires calculations of the same dpi's as would be needed to apply the single EET separately for each extra element.

As for the single EET, there are redundancy relations between the reference gains and the dpi's:

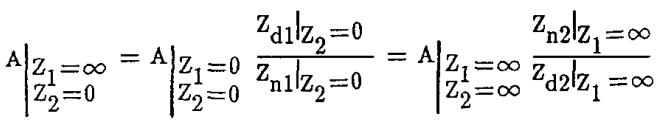

Use of the 2EET requires calculation of two new quantities, $K_{n}$ and $K_{\mathrm{d}}$, in addition to the dpi's that would be needed to apply the single EET for each extra element. These may be called interaction parameters since they are coefficients of the normalized $\mathrm{Z}_{1} \mathrm{Z}_{2}$ product terms. As seen from Eqs. (6) and (7), each interaction parameter contains one dpi already defined, and one new one for the other extra element at the opposite reference value from that in the corresponding reference gain. Note the redundancies that are displayed in Eqs. (6) and (7) that result from the subscript symmetry.

The significance of the interaction parameters $K_{n}$ and $K_{d}$ is the following. If there is no interaction between the extra elements $Z_{1}$ and $Z_{2}$, the dpi's seen by one element are independent of the value of the other element, that is, independent of whether the other element is zero or infinite. Therefore, from Eqs. (6) and (7), $K_{n}=1$ and $\mathrm{K}_{\mathrm{d}}=1$. Consequently, the numerator and denominator of the $2 \mathrm{EET}$ correction factor are exactly factorable. In the case of the 2EET version of Eq. (3), the result is

$$
\left.\underset{\mathrm{K}_{\mathrm{d}}=1}{\mathrm{~A}=\mathrm{A}=1}\right|_{\substack{\mathrm{Z}_{1}=\infty \\ \mathrm{Z}_{2}=\infty}} \frac{1+\frac{\mathrm{Z}_{\mathrm{n} 1} \mathrm{Z}_{2}=\infty}{\mathrm{Z}_{1}}}{1+\frac{\mathrm{Z}_{\mathrm{d} 1} \mathrm{Z}_{2}=\infty}{\mathrm{Z}_{1}}} \frac{1+\frac{\mathrm{Z}_{\mathrm{n} 2} \mathrm{Z}_{1}=\infty}{\mathrm{Z}_{2}}}{1+\frac{\mathrm{Z}_{\mathrm{d} 2} \mathrm{Z}_{1}=\infty}{\mathrm{Z}_{2}}}
$$

This is the same result as would be obtained by successive use of the single EET for each element, which is to be expected since, if there is no interaction between $\mathrm{Z}_{1}$ and $\mathrm{Z}_{2}$, the dpi's for each are independent of whether the other element is present or not.

As a final comment on the structure and interpretation of the 2EET, it may be pointed out that the numerator and denominator of the correction factor are always of the same format, the only difference being that the "sub $d$ " quantities in the denominator are calculated under single injection conditions, and the "sub $n$ " quantities in the numerator are calculated under null double injection conditions. Nevertheless, even if in a particular case one interaction parameter is unity, the other may not be. In fact, it is not uncommon to have $K_{n}=1$, but $K_{d} \neq 1$, so that the numerator would factor exactly into separate $\mathrm{Z}_{1}$ and $\mathrm{Z}_{2}$ terms, but the denominator would not.

Any version of the 2EET of course applies to any transfer function including self-impedances such as input and output impedances.

\section{Application of the 2EET}

The principal benefit of the 2EET is that a required transfer function is presented in a Low-Entropy Expression that is assembled, essentially in final form, from several separate calculations on a reference circuit that is simpler by the absence of two elements. This is usually a considerable improvement over the conventional derivation based on the complete circuit.

Low-Entropy Expressions are the key to Design-Oriented Analysis, which is the only analysis worth doing [2]. The particular benefit is that the format of the result can be consciously selected to expose the features that are relevant to the design choices to be made.

An example will illustrate many of the advantages resulting from use of the 2EET in the context of Design-Oriented Analysis.

Consider the bridged-T filter of Fig. 2. The objective is to find the voltage transfer function $\mathrm{A}$, and to select element values that will

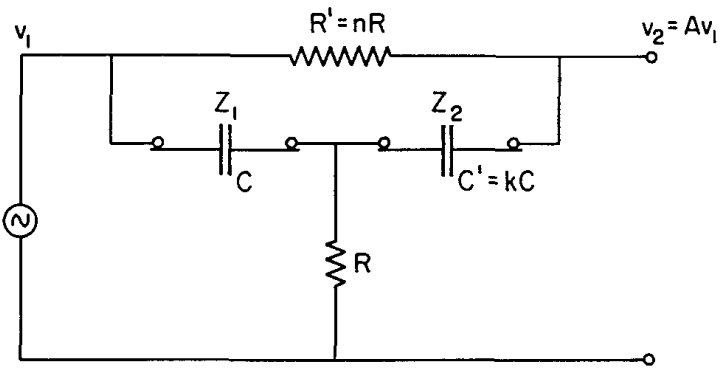

Fig. 2. The bridged-T filter: an example for application of the $2 E E T$.

produce a given specification for this transfer function. It may be anticipated that lower-entropy expressions will be obtained if normalized element values are used; therefore, let $R^{\prime}=n R$ and $\mathrm{C}^{\prime}=\mathrm{kC}$, so that the four elements are represented by $\mathrm{R}, \mathrm{C}, \mathrm{n}$, and $\mathrm{k}$.

Two of the circuit elements in Fig. 2 may be designated as "extra" elements in order to invoke the $2 \mathrm{EET}$ as an analysis tool. The obvious choice is the two reactances $C$ and $C^{\prime}$ for $Z_{1}$ and $Z_{2}$, since then the rest of the circuit, the reference circuit, is resistive and the $2 \mathrm{EET}$ correction factor will immediately expose the frequency response.

\section{Frontiers in Education Conference}


The next choice is which form of the 2EET to adopt, which translates to which reference gain we want to select. Since both extra elements are capacitances, $Z_{1}$ and $Z_{2}$ are both infinite at zero frequency, and zero at infinite frequency; therefore, $\left.A\right|_{1}=\infty$ corresponds to the zero-frequency gain, $A \cdot \mid \begin{aligned} & z_{1}=0 \\ & z_{2}=0\end{aligned}$

to the infinte frequency gain of the circuit, while $\mathrm{A} \mid z_{1}=\infty$ does not have a direct interpretation.

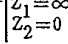

Let us choose the zero-frequency gain $A \cdot \mid \begin{aligned} & z_{1}=\infty \\ & z_{2}=\infty\end{aligned}$ as reference,

so that the appropriate 2EET form is Eq. (3) whose correction factor will give the frequency response relative to that at zero frequency. The reference circuit is therefore that in Fig. 3, upon which all of the

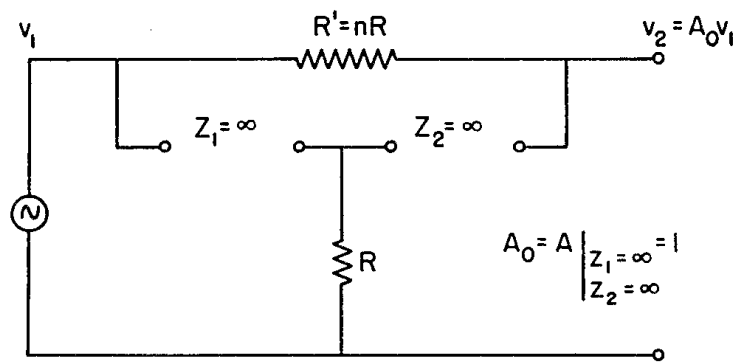

Fig. 3. The reference circuit for Fig. 2: the two capacitances are designated as "extra" elements with infinite reference impedances (opens), so that the reference gain is the zera-frequency gain of the original filter.

necessary calculations to assemble the result for the transfer function are performed. Note that this circuit is simpler than the original circuit of Fig. 2 by the absence of two of the elements. The zerofrequency reference gain is obviously unity, and is noted directly on Fig. 3.

A total of eight dpi's have been identified: the single-injection ("sub d") and null double injection ("sub n") dpi's for each extra element with the other extra element at its reference value (infinite in this case), and the same for each extra element with the other extra element at its opposite of the reference value (zero in this case) Because of the redundancy relations of Eqs. (6) and (7), only six of these need be calculated for substitution into the 2EET.

However, rather than to select in advance which six to calculate, it is usually easier just to calculate all eight, preferably in some ordered sequence, and then to confirm that the redundancy relations are satisfied as a check that no error was made.

The conditions for the calculation of the four dpi's for the extra element $Z_{1}$ are shown in Fig. 4 , and for the four dpi's for $Z_{2}$ in Fig. 5. In each case, the calculation is simple enough to be done by inspection, and the result is noted on each diagram. Familiarity with the calculation of the null double injection dpi's may be gained from [1].

Substitution of the dpi results from Figs. 4 and 5 into Eqs. (6) and (7) confirms the redundancy relations and gives

$$
\begin{aligned}
& \mathrm{K}_{\mathrm{n}}=\mathrm{n} \\
& \mathrm{K}_{\mathrm{d}}=\frac{\mathrm{n}}{\mathrm{n}+1}
\end{aligned}
$$

It remains only to substitute the reference gain, the dpi's, and the interaction parameters into the chosen version of the $2 \mathrm{EET}$ together with restoration of the extra elements $Z_{1}=1 / \mathrm{sC}$, $\mathrm{Z}_{2}=1 / \mathrm{skC}$ to give

$$
A \frac{1+s(1+k) C R+s^{2} k n C^{2} R^{2}}{1+s[1+k(n+1)] C R+s^{2} k n C^{2} R^{2}}
$$

This is the voltage transfer function of the original bridged-T circuit of Fig. 2, and the 2EET has now served its purpose. The result is a biquadratic in complex frequency s, and we next preserve the LowEntropy benefits of the result by identifying suitable corner frequencies and $Q$-factors to represent the quadratics, which gives

$$
A=\frac{1+\frac{1}{Q_{n}}\left(\frac{s}{\omega_{0}}\right)+\left(\frac{s}{\omega_{0}}\right)^{2}}{1+\frac{1}{Q_{d}}\left(\frac{s}{\omega_{0}}\right)+\left(\frac{s}{\omega_{0}}\right)^{2}}
$$

where

$$
\begin{aligned}
& \omega_{0} \equiv \frac{1}{C R \sqrt{k n}} \\
& Q_{n} \equiv \frac{\sqrt{k n}}{1+k} \\
& Q_{d} \equiv \frac{\sqrt{k n}}{1+k(n+1)}
\end{aligned}
$$

The corner frequency $\omega_{0}$ is the same for both quadratics, since for this circuit the infinite-frequency and zero-frequency gains are the same.

The biquadratic form of Eq. (13) indicates that the transfer function $A$ is symmetric about $\omega_{0}$, at which frequency $A=Q_{d} / Q_{n}$. It is obvious from Eqs. (15) and (16) that $Q_{d}$ is always less than $Q_{n}$ so the circuit is necessarily a band injection filter. Further inspection shows that $Q_{d}<0.5$ regardless of $n$ and $k$, so the two poles of the transfer function are always real. This limitation would have been much harder to divine if $Q_{d}$ had been expressed in terms of the original four circuit elements, instead of their ratios $n$ and $k$; this is the benefit of introducing such normalizing quantities in the first place. Since $Q_{n}$ can have any value, the zeros of the transfer function can be either real or complex.

Hence, the Low-Entropy results of Eqs. (13) through (16) show that the shape of the filter transfer function is constrained by the following inequalities:

$$
\mathrm{Q}_{\mathrm{n}}>\mathrm{Q}_{\mathrm{d}}<0.5
$$

The purpose in obtaining the result of Eq. (13) is to design the filter to meet a Specification that is equivalent to a required set of values for the parameters $\omega_{0}, Q_{n}$, and $Q_{d}$, which of course must satisfy the inequalities of (17) for the design to be possible. Since there are four elements in the circuit but only three parameters, the value of one element is arbitrary (it sets the impedance level). Design is the reverse of analysis [2], and so the design procedure in this example is to "invert" the analysis results of Eqs. (14) through (16): that is, $n$ and $k$ are solved from Eqs. (15) and (16), and then the CR product is found from Eq. (14). Since one element is arbitrary, the remaining one is thereby obtained.

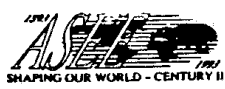

1991 Frontiers in Education Conference

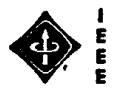




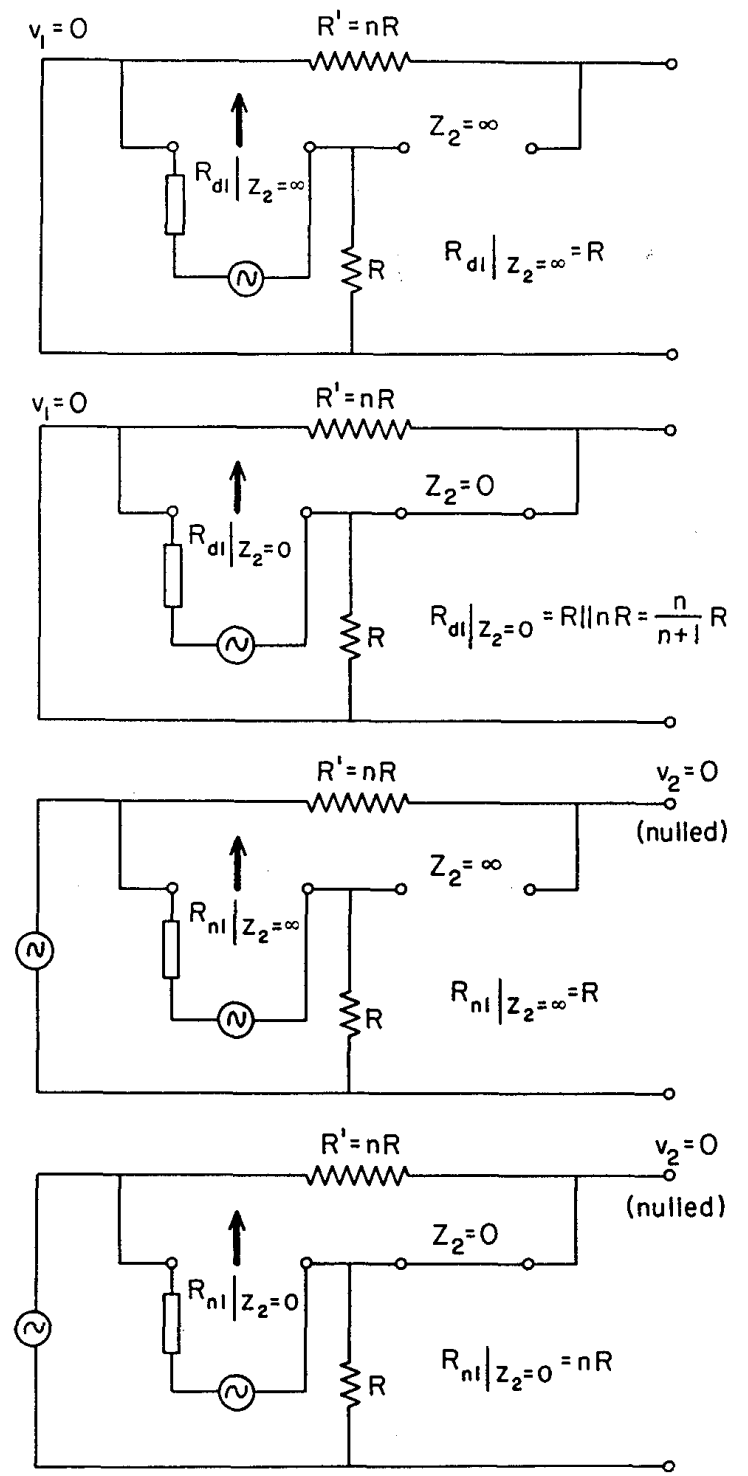

Fig. 4. Conditions upon the reference circuit of Fig. 2 for calculations of the four dpi's seen by element $Z_{1}$.

\section{Conclusions}

The single Extra Element theorem is reviewed. One of its useful applications is for determination of a transfer function by assembly of the results of calculations upon a simpler circuit model in which one element is absent. The Two Extra Element theorem is a logical extension of the single EET.

The 2EET offers a means for determination of a linear circuit model transfer function by assembly of the results of calculations solely upon a reference circuit model that is simpler by the absence of two elements.

1991 Frontler

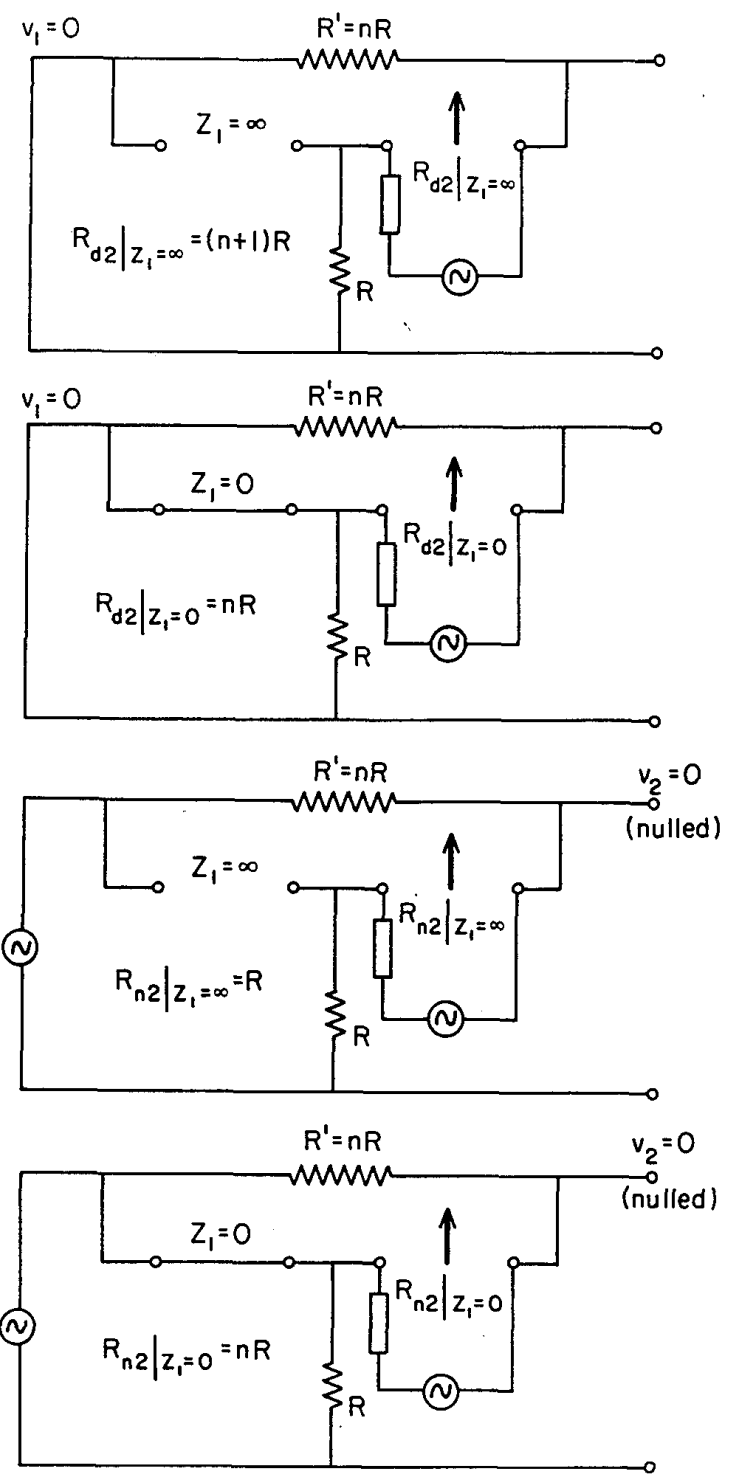

Fig. 5. Same as Fig. 4, but for calculation of the four dpi's seen by element $Z_{2}$.

Figure 1 shows a linear system model with a transfer function represented by the voltage gain $A$. To apply the 2 EET, two elements $Z_{1}$ and $Z_{2}$ are designated as "extra," and a reference gain is calculated for the reference circuit in the absence of $Z_{1}$ and $Z_{2}$, that is, with $Z_{1}$ and $Z_{2}$ replaced either by an open or a short. Since there are three nonredundant combinations of $Z_{1}$ and $Z_{2}$ open or short, there are three reference gains, and three versions of the 2EET given in Eqs. (3) through (5).

In each version of the 2EET, the reference gain is multiplied by a correction factor that involves, symmetrically, the two "extra" elements $Z_{1}$ and $Z_{2}$, and six parameters that are each calculated 
from the reference circuit. Four of these are driving point impedances, two in the denominator ("sub d") of the 2EET correction factor, calculated under single injection conditions, and two in the numerator, ("sub $\mathrm{n}^{\text {") }}$, calculated under null double injection conditions.

The two remaining parameters are the interaction parameters $K_{n}$ and $K_{d}$, Eqs. (6) and (7), requiring calculation of two additiona dpi's, those for the other "extra" element having a value opposite from its reference value. If there is no interaction between the two "extra" elements, the dpi's for one element are independent of the value of the other element, which makes $K_{n}$ and/or $K_{d}$ equal to unity. In turn, this permits the numerator and/or the denominator of the 2EET correction factor to be factored exactly, as in Eq. (9), for example, which is the same result that would be obtained by applying the single EET successively for each extra element.

There are redundancy relationships between the various reference gains and dpi's, expressed in Eqs. (6) through (8).

The 2EET provides not only a potentially shorter, simpler, and easier analysis method than solution of the transfer function directly on the complete circuit, but it also constitutes a Low-Entropy Expression. That is, the structure of the 2EET correction factor reveals additional information helpful in design, since it exposes the nature of the influence of each "extra" element normalized to an appropriate dpi

The Low-Entropy form is particularly simple and illuminating when the two designated "extra" elements are reactances and the reference circuit is resistive, since then each normalized "extra" element term becomes an " $\mathrm{s}$ " term (complex frequency), and the numerator and denominator of the 2EET correction factor each becomes a quadratic in $\mathbf{s}$.

This case is illustrated by analysis of a bridged-T filter by use of the $2 \mathrm{EET}$, in which the resulting biquadratic voltage transfer function is immediately expressed in terms of the design-oriented parameters corner frequency and Q-factors. Thus, the filter transfer function is assembled in design-oriented, Low-Entropy form with almost no algebraic manipulation at all.

The 2EET, like the single EET, can be applied to any transfer function of a linear system, including a self-impedance such as input or output impedance.

Although not mentioned here, the 2EET, as for the single EET discussed in [1], can also be applied when one or both of the "extra" elements are dependent generators.

\section{$\underline{\text { References }}$}

[1] R. D. Middlebrook, "Null Double Injection and the Extra Element Theorem," IEEE Trans. on Education, vol. 32, no. 3, August 1989, pp. 167-180.

[2] R. D. Middlebrook, "Low-Entropy Expressions: the Key to Design-Oriented Analysis," these Proceedings.

[3] R. D. Middlebrook, "Input Filter Considerations in Design and Application of Switching Regulators," IEEE Industry Applications Society Annual Meeting, 1976 Record, pp. 366-382.

\section{Appendix \\ Derivation of the 2EET}

The 2EET is obtained by application of the single EET twice in succession. In Fig. 1, suppose the reference condition is $\mathrm{Z}_{1}=\infty$, $\mathrm{Z}_{2}=\infty$. Then if the extra elements are added in the order $\mathrm{Z}_{1}$ first, $\mathrm{Z}_{2}$ second, the system gain is

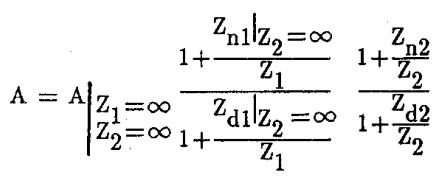

This is not the desired result since $Z_{1}$ is implicit in $Z_{d 2}$ and $Z_{n 2}$, because $Z_{1}$ is already present when these dpi's are calculated prior to addition of $\mathrm{Z}_{2}$.

The objective is to express $\mathrm{Z}_{\mathrm{d} 2}$ and $\mathrm{Z}_{\mathrm{n}}$ explicitly in terms of $\mathrm{Z}_{1}$. This can be done by identifying $\mathrm{Z}_{\mathrm{d} 2}$ and $\mathrm{Z}_{\mathrm{n}}$ as additional transfer functions of the system in the presence of $Z_{1}$ and absence of $\mathrm{Z}_{2}$, and using the single EET (again) to express these transfer functions in terms of their values $Z_{1} / z_{1}=\infty$ and $Z_{n} / z_{1}=\infty$ when $\mathrm{Z}_{1}$ is infinite. That is, the single EET is used in a "nested" fashion, to calculate $\mathrm{Z}_{\mathrm{d} 2}$ and $\mathrm{Z}_{\mathrm{n} 2}$ as part of the successive application of the single EET to find the system gain in the presence of both $Z_{1}$ and $\mathrm{Z}_{2}$.

It is important to identify properiy the transfer functions and the conditions under which the dpi's are to be calculated. The model under consideration is Fig. 1 with $Z_{1}$ in place, but $Z_{2}$ absent and replaced by an independent current source $i$, across which appears a voltage v.

The first objective is to find $\mathrm{Z}_{\mathrm{d} 2}=\mathrm{v} / \mathrm{i}$ where $\mathrm{Z}_{\mathrm{d} 2}$ is a selfimpedance transfer function whose (single-injection) "input" is $i$ and whose "output" is $v$. By the single EET, $\mathrm{z}_{\mathrm{d} 2}$ can be written explicitly in terms of $Z_{1}$ as

$$
\mathrm{Z}_{\mathrm{d} 2}=\mathrm{Z}_{\mathrm{d} 2} \mathrm{Z}_{1}=\infty \frac{1+\frac{\mathrm{Z}_{\mathrm{N}}}{\mathrm{Z}_{1}}}{1+\frac{\mathrm{Z}_{\mathrm{D}}}{\mathrm{Z}_{1}}}
$$

where $Z_{N}$ and $Z_{D}$ are appropriate dpi's seen by $Z_{1}$.

As discussed in [1], a self-impedance is a special case in which setting the input to zero is equivalent to opening the input, and nulling the output is the same as shorting the input. Here, the "input" is $i$, so opening the input means $Z_{2}=\infty$; the "output" is $v$, so shorting the input means $\mathrm{Z}_{2}=0$. Hence, $\mathrm{Z}_{\mathrm{D}}=\mathrm{Z}_{\mathrm{d} 1} \mathrm{Z}_{\mathrm{Z}_{2}}=\infty$ and $\mathrm{Z}_{\mathrm{N}}=\left.\mathrm{Z}_{\mathrm{d} 1}\right|_{\mathrm{Z}_{2}}=0$. Thus, Eq. (A.2) becomes

$$
\mathrm{z}_{\mathrm{d} 2}=\mathrm{z}_{\mathrm{d} 2} \mathrm{z}_{1}=\infty \frac{\mathrm{z}_{\mathrm{d} 1} \mathrm{z}_{2}=0}{1+\frac{\mathrm{z}_{1}}{1+\frac{\mathrm{z}_{\mathrm{d} 1} \mathrm{z}_{2}=\infty}{\mathrm{z}_{1}}}}
$$

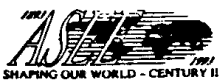

1991 Frontiers In Education Conference 707 
The second objective is to find $Z_{n 2}=v / i$ where $Z_{n 2}$ is a selfimpedance transfer function whose (nulf-double injection) "inputs" are $v_{1}$ and $i$, and whose "output" is v. The procedure is exactly the same as above for $Z_{d 2}$, except with "sub $n$ " replacing "sub d." The result is

$$
\mathrm{z}_{\mathrm{n} 2}=\mathrm{z}_{\mathrm{n} 2} \mathrm{Z}_{1}=\infty \frac{1+\frac{\mathrm{Z}_{\mathrm{n} 1} \mathrm{Z}_{2}=0}{\mathrm{Z}_{1}}}{1+\frac{\mathrm{Z}_{\mathrm{n} 1} \mathrm{Z}_{2}=\infty}{\mathrm{Z}_{1}}}
$$
gives

It remains to substitute Eqs. (A.3) and (A.4) into (A.1), which

$$
\mathrm{A}=\mathrm{A} \mid \mathrm{Z}_{\mathrm{Z}=\infty} \frac{1+\frac{\mathrm{Z}_{\mathrm{n} 1} \mathrm{Z}_{2}=\infty}{\mathrm{Z}_{1}}+\frac{\mathrm{Z}_{\mathrm{n} 2} \mathrm{Z}_{1}=\infty}{\mathrm{Z}_{2}}+\mathrm{K}_{\mathrm{n}} \frac{\mathrm{Z}_{\mathrm{n} 1} \mathrm{Z}_{2}=0}{\mathrm{Z}_{1}} \frac{\mathrm{Z}_{\mathrm{n} 2} \mathrm{Z}_{1}=\infty}{\mathrm{Z}_{2}}}{1+\frac{\mathrm{Z}_{\mathrm{d} 1} \mathrm{Z}_{2}=\infty}{\mathrm{Z}_{1}}+\frac{\mathrm{Z}_{\mathrm{d} 2} \mathrm{Z}_{1}=\infty}{\mathrm{Z}_{2}}+\mathrm{K}_{\mathrm{d}} \frac{\mathrm{Z}_{\mathrm{d} 1} \mathrm{Z}_{2}=0}{\mathrm{Z}_{1}} \frac{\mathrm{Z}_{\mathrm{d} 2} \mathrm{Z}_{1}=\infty}{\mathrm{Z}_{2}}}
$$

This is essentially the 2 EET for the reference condition $\mathrm{Z}_{1}=\infty$, $\mathrm{Z}_{2}=\infty$. Note however that while $\mathrm{Z}_{2}$ is normalized to dpl's with $\mathrm{Z}_{1}^{2}=\infty$ in all four positions, $\mathrm{Z}_{1}$ is normalized in two positions to dpi's with $\mathrm{Z}_{2}=0$, the opposite from the reference condition. To enforce normalization symmetry, the parameters $K_{n}$ and $K_{d}$ are

$$
\begin{gathered}
\mathrm{K}_{\mathrm{n}} \equiv \frac{\mathrm{z}_{\mathrm{n} 1} \mathrm{Z}_{2}=0}{\mathrm{Z}_{\mathrm{n} 1} \mathrm{Z}_{2}=\infty} \\
\mathrm{K}_{\mathrm{d}} \equiv \frac{\mathrm{z}_{\mathrm{d} 1} \mathrm{z}_{2}=0}{\mathrm{Z}_{\mathrm{d} 1} \mathrm{Z}_{2}=\infty}
\end{gathered}
$$

which puts Eq. (A.5) into the final form of Eq. (3).

In the above derivation, the 2EET was developed by adding first $Z_{1}$, and then $Z_{2}$ to the reference circuit. Of course, the result must be the same if the extra elements are introduced in the reverse order, which would be equivalent to the same derivation but with interchanged subscripts. Hence, the definitions of $\mathrm{K}_{\mathrm{n}}$ and $\mathrm{K}_{\mathrm{d}}$ in Eqs. (A.6) and (A.7) can also be expressed with interchanged subscripts, which leads to the redundant definitions of Eqs. (6) and (7).

In some applications, one or another of the dpi's may be zero, infinite, or indeterminate, and it may be preferable to avoid the interaction parameters $K_{n}$ or $K_{d}$, and to use Eq. (A.5) directly.

The other two versions of the 2EET in Eqs. (4) and (5) are obtained by manipulation of Eq. (3), with use of the redundancy ratios from Eqs. (6) and (7).

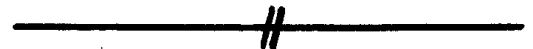

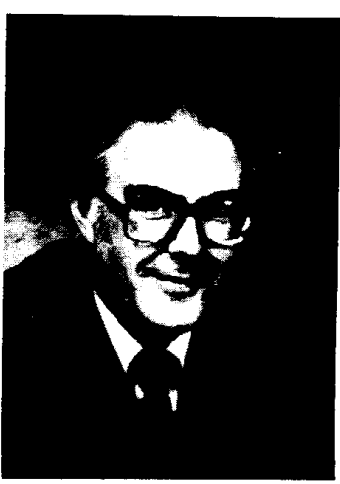

\section{R.D. MIDDLEBROOK}

received the B.A. and M.A. degrees from Cambridge University, England, and the M.S. and Ph.D. degrees from Stanford University, Stanford, CA, in 1952, 1954, 1953, and 1955, respectively.

$\mathrm{He}$ is Professor of Electrical Engineering at the California Institute of Technology (Caltech). His publications include numerous papers, a book on solid-state device theory, and another on differential amplifiers. His research interests, formerly in solid-state device modeling, are now in circuits and systems, and particularly in power processing electronics in which he is well-known as author and lecturer. $\mathrm{He}$ is especially interested in design-oriented circuit analysis and measurement techniques which he teaches at Caltech, and he has conducted short courses on his methods in both Europe and the United States.

Dr. Middlebrook is the recipient of the 1982 IEEE William E. Newell Power Electronics Award for

Outstanding Achievement in Power Electronics, and a 1982 Award for Excellence in Teaching, presented by the Board of Directors of the Associated Students of Caltech. In 1984 he received an IEEE Centennial Medal, and in 1991 he was awarded the Edward Longstreth Medal of the Franklin Institute.
1991 Frontlers in Education Conference

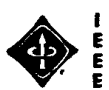

